A N N A LES

UNIVERSITATIS MARIAE CURIE-SKŁODOWSKA

LUBLIN - POLONIA

VOL. LXVI, 1, 2

\title{
Synthesis and characterization of $\mathrm{Pt} / \mathrm{CeO}_{\mathrm{x}}$ systems for catalytic $\mathrm{CO}$ oxidation reaction*
}

\author{
R. J. Wróbel ${ }^{1 *}$, S. Łagonda ${ }^{1}$, U. Narkiewicz ${ }^{1}$, R. Pełech ${ }^{2}$, S. Becker ${ }^{3}$, \\ L. Szymaszkiewicz ${ }^{4}$ \\ ${ }^{1,2}$ West Pomeranian University of Technology, ${ }^{1}$ Institute of Chemical and \\ Environment Engineering, ${ }^{2}$ Institute of Organic Chemical Technology, \\ Pulaskiego 10, 70-322 Szczecin, Poland \\ ${ }^{3}$ Otto-von-Guericke Universität, Chemisches Institut, Universitätsplatz, 2, \\ 39106 Magdeburg, Germany \\ ${ }^{4}$ Szczecin University, Institute of Mathematics, \\ Wielkopolska 15, 70-451 Szczecin, Poland
}

\begin{abstract}
Four different ceria supported catalyst were prepared by impregnation method with $\mathrm{Pt}\left(\mathrm{NO}_{3}\right)_{2}$ solution. The two supports are commercially available (MaTeck) and the other two were prepared by precipitation and microwave assisted hydrothermal method (MAH) respectively. The phase composition and average crystallite size of the catalysts were characterised with XRD technique. Finally the catalytic activity in $\mathrm{CO}$ oxidation reaction were determined in plug flow reactor in temperature range 300-900 K with $1 \mathrm{~K}$ resolution. The catalysts obtained in both precipitation and MAH methods exhibit catalytic activity at room temperature whereas catalysts obtained on MaTeck supports are not active at those conditions. In turn, catalysts based on MaTeck support are more active in temperature range 420-700 K. The different activities are attributed to difference in average crystallite sizes and in support morphology.
\end{abstract}

\footnotetext{
*This article is dedicated to Professor Dobiesław Nazimek on the occasion of his $65^{\text {th }}$ birthday

*corresponding author: rwrobel@zut.edu.pl
} 


\section{INTRODUCTION}

The CO oxidation reaction catalyzed by platinum group metals, despite many decades of intensive research, still is investigated worldwide [1-4]. The research is motivated by various reasons. On one hand the $\mathrm{CO}$ oxidation is a relatively simple reaction. Therefore enables to understand catalytic phenomena on atomic level. On the other this reaction is widely applied e.g. in industry, automotive exhaust gas abatement and purification of hydrogen for fuel cells, to mention but a few. The measure of importance of this topic can be the Nobel Prize in chemistry given in 2007 to Gerhard Ertl "for his studies of chemical processes on solid surfaces" including $\mathrm{CO}$ oxidation on platinum group metals [5].

One can argue that after so many years of research, a little left to discover. Nothing further from the truth. The phenomena discovered by G. Ertl only shows how complicated the $\mathrm{CO}$ oxidation, even on highly simplified model systems, can be. The real systems are far more complex and application of knowledge acquired on model systems are not so straightforward. Due to that in industry is usually applied different i.e. experimental approach. The catalyst systems are optimized "blindly" to acquire the highest activity, durability etc. However "blind" optimisation is very limited. The catalytic activity is a function of numerous factors. Therefore the optimisation process will rather find local, than global maximum. To overcome these difficulties the understanding on the atomic level of the phenomenon is required. Indeed many aspects of the CO oxidation were good understood by investigation of model systems [6-9]. It is commonly known that $\mathrm{CO}$ oxidation occurs according to the Langmuir-Hinshelwood mechanism [10]. The oxygen molecule to successfully react with $\mathrm{CO}$ molecule has to adsorb on the surface and then dissociate. To achieve this, a two adjacent adsorption places on platinum metal are required. This phenomenon causes that platinum surface can be easily poisoned by $\mathrm{CO}$. The poisoning process is reversible and depends on the conditions the hysteresis in reaction rate is observed [7,11,12]. The ceria support serves as a oxygen storage in oxygen lean periods of the reaction. It has been also found that ceria itself promotes $\mathrm{CO}$ oxidation reaction [7] and the spill-over of oxygen from ceria to platinum may occur [13] thus the poisoning of the platinum is limited for higher $\mathrm{CO}$ partial pressures.

Most of above mentioned phenomena were discovered with surface science approach i.e. in ultra high vacuum conditions and on well defined model catalysts. The conditions required for surface science approach are quite different than working conditions of real catalyst. Therefore there is an urge to 
bridge so called "pressure and material gaps". The goal of this paper is extension of our previous experiments to the normal pressures and powder materials.

\section{MATERIAL AND METHODS}

\subsection{Preparation of the ceria supports}

Two supports are commercially available by MaTeck: Cerium (IV) oxide nano-powder (CN 903577) $99.5 \%$ purity, specific surface area $28-40 \mathrm{~m}^{2} / \mathrm{g}$, average particle size 20-30 nm. Cerium (IV) oxide nano-powder (CN 903576) $99.9 \%$ purity, average particle size $70-105 \mathrm{~nm}$. The supports and catalysts derived from them are further named as $M 30$ and $M 70$ catalyst or support respectively.

The third support was prepared by mixing the solutions of ammonium hydroxide and $\mathrm{Ce}\left(\mathrm{NO}_{3}\right)_{3}$ (Alfa Aesar, $99.5 \%$ purity) at room temperature. Subsequently the obtained cerium hydroxide was separated from liquid in centrifugal separator, washed several times with distilled water and calcined at $900 \mathrm{~K}$ in air. The support and catalyst derived from this preparation procedure is further named as $\boldsymbol{P}$ catalyst or support.

The fourth support was prepared by mixing the solutions of ammonium hydroxide and $\mathrm{Ce}\left(\mathrm{NO}_{3}\right)_{3}$ (Alfa Aesar, $99.5 \%$ purity) at room temperature. Subsequently so obtained mixture was placed in microwave assisted hydrothermal reactor (Plasmatronika, Reaktor Nano Wizard 2001, frequency 4.6 Ghz, specific power $4 \mathrm{~W} / \mathrm{ccm}$ ). The temperature was raised up to $473 \mathrm{~K}$ and the pressure up to 20 bar. After preparation the ceria compound was separated, washed several times with distilled water and calcined at $900 \mathrm{~K}$ in air. The support and catalyst derived from this preparation procedure is further named as $M A H$ catalyst or support.

\subsection{Preparation of $\mathrm{Pt} / \mathrm{CeO}_{2}$ systems}

The calcination in air at $900 \mathrm{~K}$ enables removal of any undesired cerium compounds like carbonates and/or hydrates and decomposition of them to the ceria. This in turn enables preparation of the catalyst with the exact platinum to ceria ratio. The calcined supports were weighted up, soaked with $\operatorname{Pt}\left(\mathrm{NO}_{3}\right)_{2}$ solution (MaTeck CN 006438) with amount enabling 1\% Pt concentration after thermal nitride decomposition. The subsequent step was calcination at $900 \mathrm{~K}$ in air followed by grinding at mortar. During calcination the decomposition of $\mathrm{Pt}\left(\mathrm{NO}_{3}\right)_{2}$ occurs and the product is Pt on ceria support. 


\subsection{Catalyst characterisation}

The catalysts were characterised by powder X-ray diffraction (Philips Xpert Pro $)$. The $\mathrm{Cu} \mathrm{K \alpha}$ radiation $\left(\mathrm{K} \alpha_{1}=0.154056 \mathrm{~nm} ; \mathrm{K} \alpha_{2}=0.154439 \mathrm{~nm}\right)$ was applied. $\mathrm{K}_{\beta}$ was removed with filter while $\mathrm{K} \alpha_{2}$ with the software procedure. The X'Pert HighScore software has been used for data evaluation. The configuration of goniometer was Bragg-Brentano. The $20 \div 1352 \theta$ range was applied with 0.05 step and $3 \mathrm{~s}$ acquisition time for survey scans.

The average crystallite size was evaluated according to Scherrer formula [14] based on reflection from family of 620 planes. The shape factor 0.9 was assumed and broadening of the reflex originating solely from instrumental broadening and the size of the crystallites. The instrumental broadening was estimated by measurement of silicon reference sample for several reflexions and interpolation for ceria 620 reflex. The higher precision of measurement was applied i.e. step 0.02 and acquisition time $10 \mathrm{~s}$.

\subsection{Experimental setup}

In Figure 1 the schematic idea of the catalytic reactor is presented.

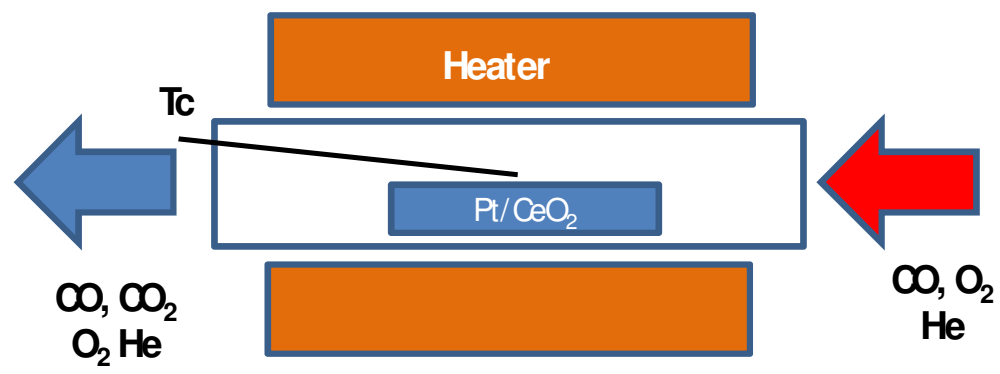

Fig. 1. The schematic representation of the catalytic reactor. The thermocouple denoted as Tc is placed just above the catalyst bed. The flow and composition of the reactant and the product is denoted with red and blue arrows respectively.

The grained sample of catalyst in amount $1.5 \mathrm{~g}$ was placed in ceramic sample holder with area of c.a. $5 \mathrm{~cm}^{2}$. Subsequently the sample holder was placed inside of quartz pipe reactor. The temperature of the sample was monitored with Pt100 thermocouple positioned just above the surface of the catalyst. The atmosphere was maintained by the constant flow of the gases $444.44 \mathrm{ccm}$ of $\mathrm{He} 2 \mathrm{ccm}$ of $\mathrm{O}_{2}$ and $4 \mathrm{ccm}$ of $\mathrm{CO}$ (Brooks instrument, Bronkhorst High-Tech, mass flow controllers). What gives the concentration of reactants and inert respectively 98.67 vol.\% of He (Messer, 5.0 purity) $0.44 \%$ of oxygen (Messer, 5.0 purity) and $0.89 \%$ carbon monoxide (Messer, 3.7 purity). The temperature was controlled by PID regulator (APAR, AR 682) with accurately tuned PID 
parameters what enables accurate $( \pm 0.1 \mathrm{~K})$ and fast temperature stabilisation. The $\mathrm{CO}$ conversion degree was monitored by $\mathrm{CO}_{2}$-IR analyser (LAB-EL, LB-850B; $0 \div 10000 \mathrm{ppm} \mathrm{CO}_{2}$ range). The linearity of the $\mathrm{CO}_{2}$-IR analyser was confirmed by the measurement of the standard gases of different $\mathrm{CO}_{2} / \mathrm{He}$ ratios. The readings of the $\mathrm{CO}_{2}$-IR analyser were independently confirmed by both, the chromatographic (Thermo Electron Corporation, Trace GC Ultra; Column: Restek, Rt-Q-BOND, $30 \mathrm{~m}, 0.53 \mathrm{~mm}$; carrier gas $\mathrm{He} 10 \mathrm{ccm} / \mathrm{min}$, isothermal measurement @ $308 \mathrm{~K}$, TCD detector) and FTIR (Thermo Electron Corporation, FT-IR Nicolet 380) measurements. All three techniques gave the same results within measurement error.

\subsection{Catalytic activity measurement}

Each catalyst, prior the catalytic activity measurement, was calcined in the reactor at $900 \mathrm{~K}$ under gas composition given above for $1 \mathrm{~h}$. Subsequently the temperature was slowly decreased with the rate $1.2 \mathrm{~K} / \mathrm{min}$ down to $300 \mathrm{~K}$. The gas composition was monitored by IR measurement with sampling rate $60 / \mathrm{min}$. The XRD measurements were performed after catalytic activity measurements.

\section{RESULTS AND DISCUSSION}

In Figure 2 are presented the XRD spectra of four catalysts i.e. M70,M30, $\mathrm{P}$ and MAH. The main phase is pure $\mathrm{CeO}_{2}$ (JCPDS 34-0394). The platinum phase (JCPDS 04-0802) is only detectable in case of M70 catalyst (see the left inset in Figure 2). The limit of XRD technique is about one weight percent. As it will be shown later the M70 sample has the highest average size of ceria crystallites, thus the specific surface area is the smallest. The platinum phase in M70 sample is the least dispersed on the surface of ceria support and can be detected by XRD method.

The right inset shows the reflex 620 utilised for determination of average crystallite sizes. It can be noticed that the catalysts differ in reflex broadening, what is a measure of crystallite size. To visualise better this effect the spectra were normalised (Figure 2 -middle inset). The increasing order of the samples based on full width at half maximum (FWHM) is as follows: M70, MAH, P and M30. This corresponds to average crystallite sizes 69, 14, 10 and $8 \mathrm{~nm}$ respectively. The average size of $69 \mathrm{~nm}$ of M70 is in a good agreement with

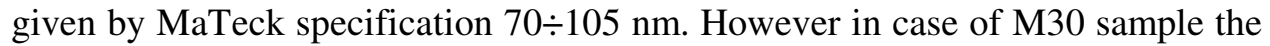
discrepancy can be noticed i.e. Obtained with Scherrer method size $8 \mathrm{~nm}$ is far less than 20-30 nm given by MaTeck. The discrepancy can be a result of different measurement method. MaTeck's information note gives the average particle size (Probably obtained with BET method) while Scherrer's formula gives average crystallite size. Please note that individual particle can consist of 
several adjacent crystallites which surface is only partially available for adsorption process, what leads to discrepancy. The other factor can be assumptions of Scherrer's method. It assumes that the broadening of the reflex is caused solely by crystallites size. In reality there can be factor originating from distribution of lattice constants. The ceria is prone to reduction process hence the oxygen vacancies can be easily formed. This process leads for broadening of the reflex, which is not related with crystallite size.

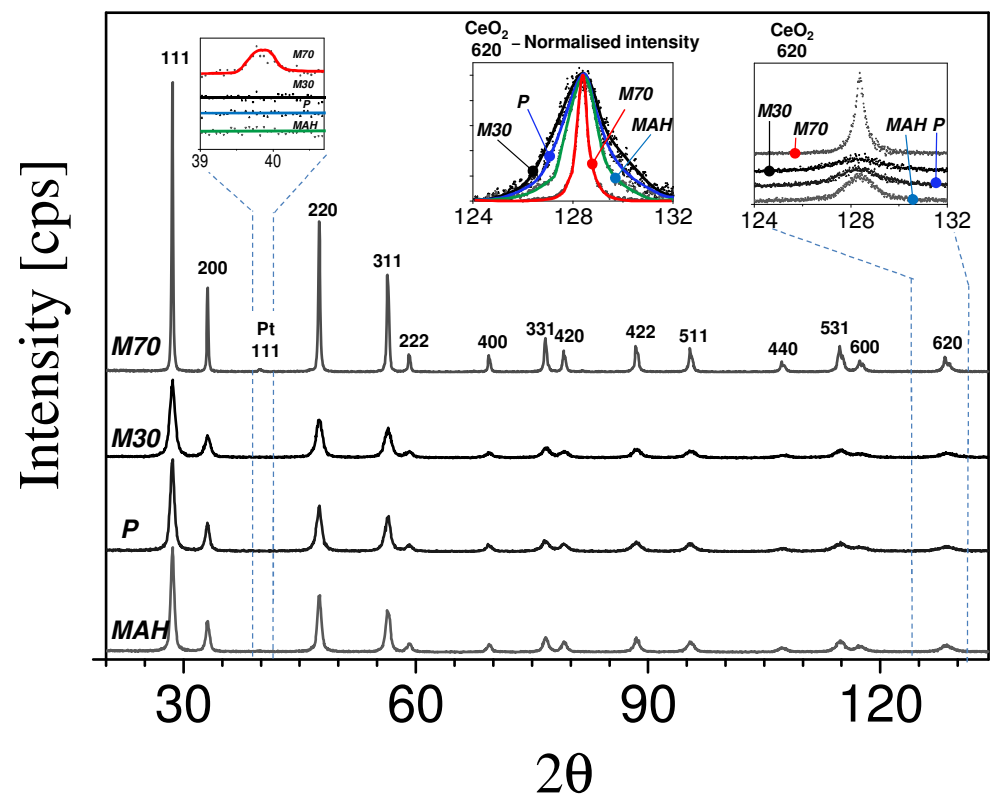

Fig. 2. The XRD spectra of investigated catalysts. The main detected phase is $\mathrm{CeO}_{2}$. In case of M70 catalyst a small reflex of Pt phase is detectable (left inset). The average crystallite size measurement was performed based on 620 peak (right inset). The normalised intensity of 620 peak (middle inset) enables visualisation of the effect of crystallite size on the broadening of the peak.

In Figure 3 is presented the set of spectra for investigated catalysts. Each curve has similar shape however the offset on temperature axis can be noticed. The catalyst M70 loses its activity as first with temperature. This is most likely related with biggest crystallite size or, in other words, the least specific surface area. However second in turn is the catalyst M30 with highest specific area. Therefore the specific surface area is only one of the factors responsible for catalytic activity of the systems. The catalyst $\mathrm{P}$ and $\mathrm{MAH}$, despite their bigger 
value of crystallite sizes in comparison with M30 catalyst, have significant catalytic activity at $300 \mathrm{~K}$. The rate of the process in upper part of the curves is probably driven by the gas diffusion in catalyst bed.

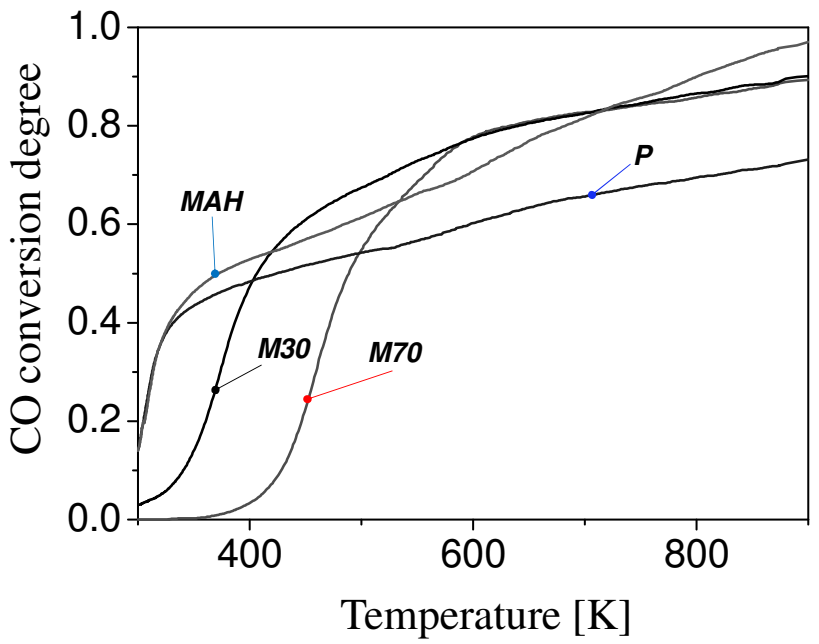

Fig. 3. The dependence of $\mathrm{CO}$ conversion degree on temperature. The most active catalyst at room temperature $(300 \mathrm{~K})$ are $\mathrm{MAH}$ and $\mathrm{P}$ catalysts respectively. The catalysts produced on MaTeck support (M30, M70) are least active on those conditions. At higher temperature range $(420 \div 700 \mathrm{~K})$ the catalyst M30, M70 are the most active catalysts. At temperatures higher than $700 \mathrm{~K}$ the most active catalyst is obtained in microwave assisted hydrothermal method (MAH).

\section{CONCLUSIONS}

The catalysts were characterised with XRD technique and their catalytic activity in $\mathrm{CO}$ oxidation reaction were measured. It was found that average crystallites size, although has impact on catalytic activity, is not the main factor responsible for activity of the catalyst. The catalysts obtained in microwave assisted hydrothermal method (MAH) and by precipitation (P) have significant catalytic activity even at room temperature, despite their larger average crystallite sizes than catalyst M30 (Pt on MaTeck support). The high activity is attributed for morphology of the catalyst. At temperature range $420 \div 700 \mathrm{~K}$ the most active catalysts are M30 and M70. At temperatures above $430 \mathrm{~K}$ the most active is MAH catalyst.

Acknowledgments. R.J.W. would like to acknowledge the support of Ministry of Science and Higher Education in the frame of the project N N209 336737. 


\section{REFERENCES}

[1] A. D. Allian, K. Kakanabe, K. L. Fujdala, X. Hao, T. J. Truex, J. Cai, C. Buda, M. Neurock, E. Iglesia, J. Am. Chem. Soc. 133, 4498 (2011).

[2] Z. Sljivancanin, B. Hammer, Phys. Rev. B 81, 121413 (2010).

[3] Y. Suchorski, C. Spiel, D. Vogel, W. Drachsel, R. Schlögl, G. Rupprechter, Chem. Phys. Chem. 11, 3231, (2010)

[4] K. Kunimatsu, T. Sato, H. Uchida, M. Watanabe, Langmuir 24, 3590 (2008).

[5] G. Ertl, Angew. Chem. Int. Ed. 47, 3524 (2008).

[6] J. A. Rodriguez, S. Ma, P. Liu, J. Hrbek, J. Evans, M. Pérez, Science 318, 1757 (2007).

[7] Y. Suchorski, R. Wrobel, S. Becker, H. Weiss, J. Phys. Chem. C 112, 20012 (2008).

[8] Y. Suchorski, R. Wrobel, S. Becker, B. Strzelczyk, W. Drachsel, H. Weiss, Surf. Sci. 601, 4843 (2007).

[9] R. Wróbel, Y. Suchorski, S. Becker, H. Weiss, Surf. Sci. 602, 436 (2008).

[10] T. Engel, G. Ertl, Adv. Catal. 28, 1 (1979).

[11] M. Berdau, Dissertation, Freie Universität Berlin (1993).

[12] R. Imbihl, G. Ertl, Chem. Rev. 95, 697 (1995).

[13] S. Becker, K. Müller, R.J. Wrobel, H. Weiss, Ceria promoted CO oxidation on Pt(111): Oxygen-spillover and active border concept, to be published.

[14] A. L. Patterson, Phys. Rev. 56, 978 (1939).

\section{CURRICULA VITAE}

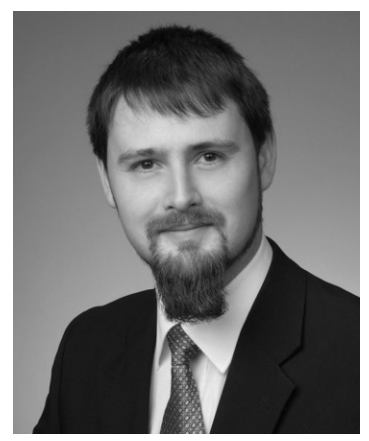

Stefan Becker. Born 04.09.1982 in Magdeburg (Germany). Graduated from Otto-von-Guericke-University of Magdeburg for Chemistry and Physics for teaching profession (2008). Ph.D. natural science (2010). Affiliation: Institute of Chemistry, Faculty of Process and Systems Engineering, Ottovon-Guericke-University of Magdeburg, Germany. Research interests: surface science, chemical reactions, nanotechnology, catalysis. Member of the German National Academic Foundation (since 2006). Awards: Grant of German National Academic Foundation (2006 - 2008), Fellowship of the Fond of Chemical Industry (2008 - 2010), Participant at the 59.th noble laureates meeting 2009 Lindau (Germany).

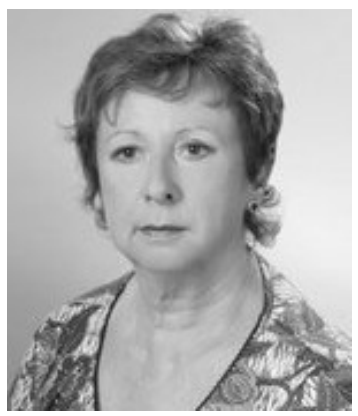

Urszula Narkiewicz. Born 11.10.1952 in Goleniów. Graduated from Szczecin University of Technology (1976). Full professor in technical sciences (2008). Affiliation: Faculty of Chemical Engineering, West Pomeranian University of Technology in Szczecin, Poland. Research interests: chemical technology, nanotechnology, catalysis. Polish expert in COST DC_MPNS Materials, Physical and Nanosciences (2006-...), Polish representative at the Mirror Group of the ETP Nanomedicine (2006-...), member of the 
Executive Committee of the European Materials Research Society (2007-...), VicePresident of the Polish Materials Science Society (2009-...), member of the Nanomaterials' Section of the Committee or the Materials Science of the Polish Academy of Science $(2007$ - ...)

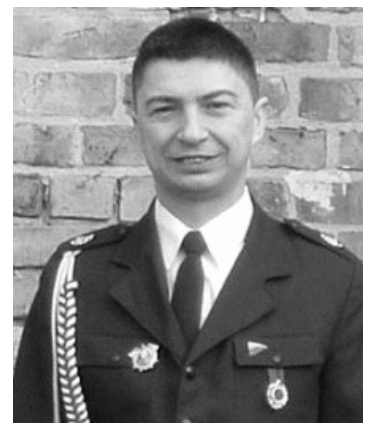

Robert Pełech. Born 22.04.1973 in Gorzów Wielkopolski. Graduated from Szczecin University of Technology (1999). $\mathrm{PhD}$ in technical sciences (2004). Affiliation: Faculty of Chemical Engineering, West Pomeranian University of Technology in Szczecin, Poland. Research interests: chemical technology, adsorption, waste utilization, catalysis. Head of Waste Utilization Laboratory. Member of OSP Nowiny Wielkie.

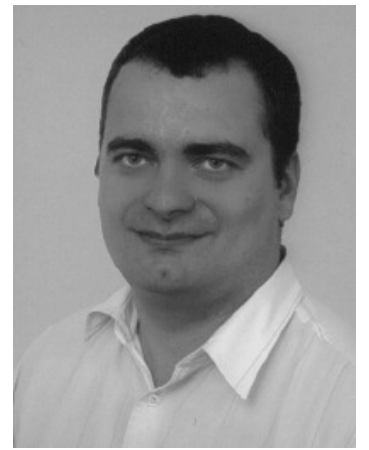

Lucjan Szymaszkiewicz was born in Szczecin on May 11, 1974. He studied at the University of Szczecin, where he received his M. Sc. degree in mathematics. He received his $\mathrm{Ph}$. D. degree in mathematics in 2005 from the Adam Mickiewicz University in Poznań. In 2009, Lucjan Szymaszkiewicz received his M. Sc. degree in computer science at the West Pomeranian University of Technology in Szczecin. Currently affiliated with the Faculty of Mathematics and Physics at the University of Szczecin. Research interests: mathematical analysis, applied mathematics, computer science.

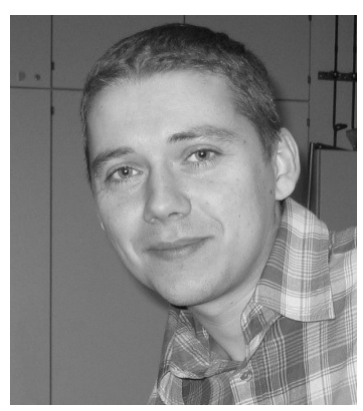

Rafał J. Wróbel was born in Szczecin on September 18, 1974. Graduated from Szczecin University of Technology (1999). Ph.D. Chemical Technology (2004) University of Technology in Szczecin. Post-doc position in Otto-vonGuericke-Universität Magdeburg, Germany (2005-2009) Research interests: chemical technology, nanotechnology, catalysis. Head of Polish-German Solid State Laboratory. His main field of interest is catalysis, gas-solid reaction, adsorption phenomena, surface science techniques, utilization of XRD technique for determination of crystallite size distribution, numerical modeling etc. 


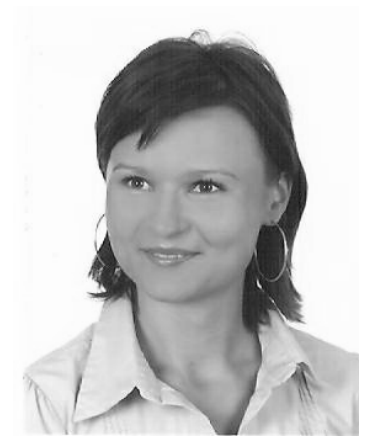

Sylwia Lagonda was born in Stargard Szczeciński on November 05, 1986. She studied at the Szczecin University of Technology, where she received her M. Sc. degree in Chemical Engineering (2010) and Engineer's degree in Science of Commodities (2009). Since October 2010 she is doctoral student of Chemical Technology at West Pomeranian University of Technology in Szczecin. Research interests: chemical technology, nanotechnology, catalysis. 